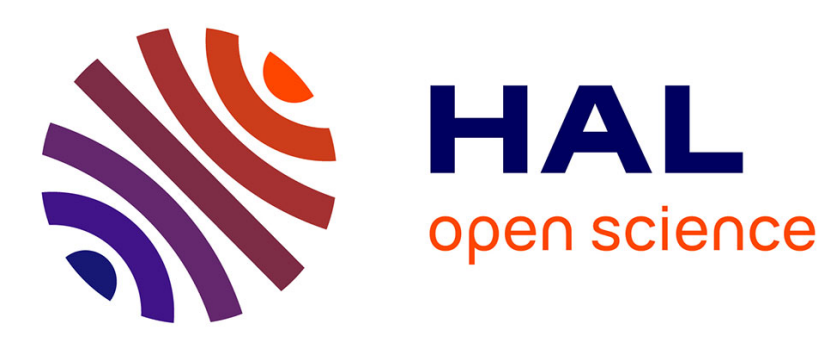

\title{
Flexible body with drag independent of the flow velocity
}

Thomas Barois, Emmanuel de Langre

\section{To cite this version:}

Thomas Barois, Emmanuel de Langre. Flexible body with drag independent of the flow velocity. Journal of Fluid Mechanics, 2013, 735, pp.R2. 10.1017/jfm.2013.516 . hal-00995147

HAL Id: hal-00995147

https://hal-polytechnique.archives-ouvertes.fr/hal-00995147

Submitted on 27 May 2014

HAL is a multi-disciplinary open access archive for the deposit and dissemination of scientific research documents, whether they are published or not. The documents may come from teaching and research institutions in France or abroad, or from public or private research centers.
L'archive ouverte pluridisciplinaire HAL, est destinée au dépôt et à la diffusion de documents scientifiques de niveau recherche, publiés ou non, émanant des établissements d'enseignement et de recherche français ou étrangers, des laboratoires publics ou privés. 


\title{
JFM RAPIDS
}

journals.cambridge.org/rapids

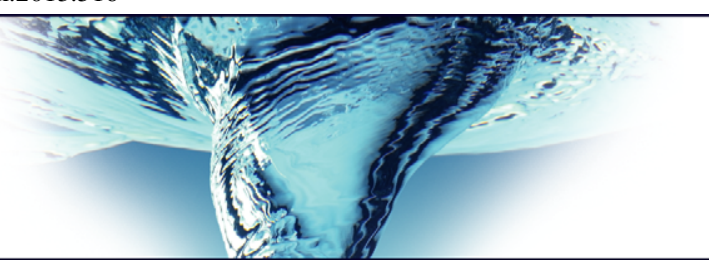

\section{Flexible body with drag independent of the flow velocity}

\author{
Thomas Barois and Emmanuel de Langre $\dagger$
}

LadHyX, Department of Mechanics, École Polytechnique, 91128 Palaiseau, France

(Received 26 July 2013; revised 17 September 2013; accepted 25 September 2013; first published online 16 October 2013)

The drag of a rigid object is expected to increase with flow velocity. For wide ranges of velocities commonly encountered, the drag increases as the square of the relative velocity of the fluid. This strong dependence of the load on the velocity accounts for specific survival strategies adopted by passive living systems such as plants in wind or algae in marine environments: through elastic reconfiguration, the drag on plants is reduced when compared to a rigid configuration and the velocity exponent for the drag is typically found to be between 1 and 1.5. In this work, a membrane configuration is presented that exhibits a drag force that is almost independent of the free-stream velocity. This surprising result is shown to be remarkably robust as it is experimentally observed for a range of geometries. This study opens the way for the design of devices subjected to a drag that is independent of the flow velocity. This possibility constitutes a key point in various fields involving flexible structures that are towed or subjected to wind.

Key words: aerodynamics, flow-structure interactions

\section{Introduction}

The observation of plants subjected to wind (Vogel 1996; Ennos 1997) has given rise to numerous studies concerning the deformation of structures in flowing fluids, an essential issue being the evolution of the drag with flow velocity. At high Reynolds numbers, the aerodynamic drag on rigid objects is known (Blevins 2003) to scale with the velocity squared. For plants, the dimensions and the wind velocities correspond to a high-Reynolds-number régime. However, the dependence of the drag on the velocity is modified because these objects are not rigid. This is also observed in aquatic environments (Sand-Jensen 2003; Statzner et al. 2006; Luhar \& Nepf 2011). Unexpectedly, the forces applied on such flexible objects are simply described by scaling laws (Harder et al. 2004; Vogel 2009) where the velocity exponent of 2 for the rigid equivalent is reduced by the value of the Vogel exponent. Previous works on the elastic reconfiguration of elementary structures (Alben, Shelley \&

$\dagger$ Email address for correspondence: delangre@ladhyx.polytechnique.fr 


\section{T. Barois and E. de Langre}

Zhang 2002; Schouveiler \& Boudaoud 2006; Gosselin, de Langre \& Machado-almeida 2010) have shown that the complexity of the fluid-structure interaction is simplified because of the existence of a Cauchy number (de Langre 2008). This dimensionless number characterizes the relative importance of fluid forces compared to the elastic counterpart.

This paper considers whether it is possible to have a deformable object for which the drag is independent of the velocity and this for a large range of experimental conditions.

In the context of fluid dynamics at high Reynolds numbers, the existence of a drag independent of the flow velocity is intriguing. It should however be mentioned that a drag independent of the velocity has been observed at low Reynolds numbers in DNA molecules (Wirtz 1995). The same behaviour is observed for objects moving within a granular medium (Albert et al. 1999) because of the grain friction law.

The approach of this work is to consider the case of membranes. It is illustrated by measuring the force applied on a weighted flexible ribbon which is towed in a water tank. When the velocity is large enough, the force needed to tow the ribbon displays a nearly horizontal plateau associated with a constant drag. This régime is shown to remain valid as long as the bending stiffness is negligible. The experiments are accompanied by a theoretical model describing the drag dependence with the velocity and also the ribbon profile.

This work focuses on the cancellation of the velocity dependence rather than on the minimization of the drag at a given velocity. In practice, a weak dependence of the drag on velocity is desirable for the towing of underwater systems in which the depth of the towed object comes from the balance between weight and drag. A similar process arises for the refuelling of aircrafts in flight in which the stability of the boom of the tanker aircraft might be affected by velocity fluctuations.

\section{Reconfiguration of membranes}

\subsection{Concept}

At high Reynolds numbers, the cross-sectional area of an object perpendicular to the incoming flow results in a force exerted by the fluid, which is associated with mass and momentum transfer both increasing linearly with velocity. Reconfiguration occurs when the shape and so the cross-sectional area of an object are modified by the oncoming stream.

Reconfiguration associated with the balance of fluid forces and bending stiffness has been widely studied. In such elastic structures, the reconfiguration régime still corresponds to an increase of the drag with velocity.

In this work, a class of deformable bodies without bending stiffness, such as membrane ribbons, is considered. The interesting property of membranes is that they transmit only the in-plane forces. The drag of a membrane is then bounded by its in-plane tension magnitude. Another property of the membrane ribbons is that they are mainly subjected to pressure drag, i.e. to normal forces. As a consequence, the tension magnitude is not modified by the fluid flow. As illustrated in the inset in figure 1 $(a)$, the fluid forces modify only the in-plane tension orientation and therefore the ribbon shape. If the tension comes from a non-fluid force such as a weighted load at one end of the ribbon, the magnitude of the drag is expected to saturate with increasing values of the upstream flow velocity. 
(a)

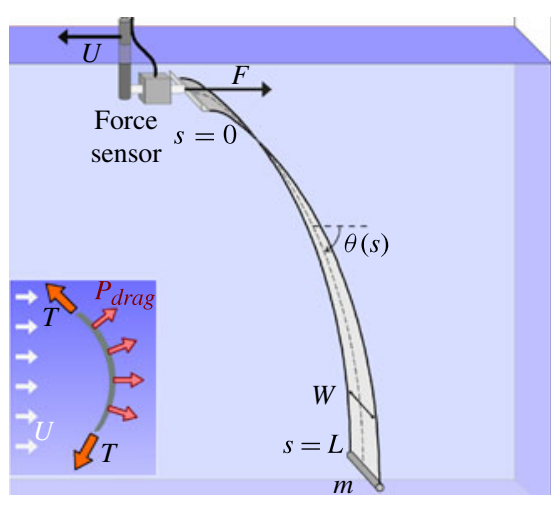

(b)

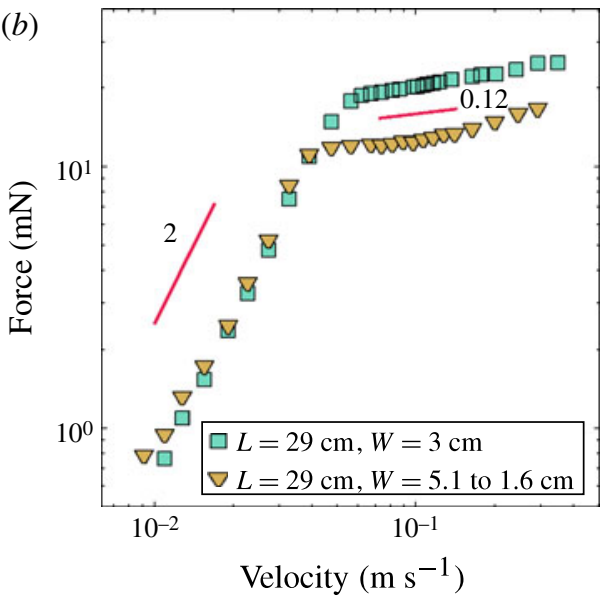

Figure 1. (a) Perspective view of the experiment in water for a rectangular ribbon of length $L$ and width $W$. The ribbon is attached to a cylinder that is towed at a constant velocity $U$ and a mass $m$ is placed at the free end. The curvilinear coordinate along the ribbon $s$ and the local angle with respect to the horizontal $\theta$ are indicated on the sketch. The force $F$ necessary to move the ribbon is measured with a sensor placed between the ribbon support and the moving cylinder. Inset: schematic of a ribbon without flexural rigidity (membrane) in a fluid flow. The fluid exerts a local pressure $P_{\text {drag }}$ on the membrane at a given velocity $U$. Two tangential forces of equal magnitude $T$, independent of $P_{\text {drag }}$, are applied to maintain the ribbon. $(b) \log -\log$ plot of the drag versus velocity measurements for a rectangular ribbon and a trapezoidal ribbon (dimensions specified in the legend). At low velocities, the drag increases with the velocity squared. At higher velocities, the drag saturates on a quasi-horizontal plateau. $\operatorname{Re}>3 \times 10^{2}$, mass attached to the free end is $2.0 \mathrm{~g}$ for the rectangular ribbon, $1.2 \mathrm{~g}$ for the trapezoidal ribbon.

\subsection{Experimental setup}

The experimental demonstration of the constant drag concept $(\S 2.1)$ has been investigated in a water tank (figure $1 a$ ). The in-plane tension is produced by a weight $m g$ which is added at the free end of the ribbon. A cylindrical rod supporting the weighted ribbon is towed at a constant velocity $U$. The force $F$ applied at the clamped end of the membrane is measured with a Futek piezoelectric sensor (figure $1 b$ ). Two membrane geometries have been considered to demonstrate the robustness of the concept. The first one is a rectangular ribbon (length $L=29 \mathrm{~cm}$, width $W=3 \mathrm{~cm})$ and the second one is a trapezoid $(L=29,5.1 \mathrm{~cm}$ for the large base and $1.6 \mathrm{~cm}$ for the short base). The membranes are attached to a small rigid support (with cross-sectional area $\mathscr{A}<1 \mathrm{~cm}^{2}$ ) and weighted with cylindrical rods with a density close to eight times that of water. The density of the ribbon is close to the density of the surrounding water. The trapezoidal ribbon is attached at its longer base. The part of the drag acting on the rigid support only is measured without the presence of the ribbon and subtracted from the force measurements with ribbon. The ribbons are attached horizontally to the rigid support in order to minimize the effect of the bending stiffness. The finite value of the bending stiffness is discussed in $\S 5$.

For both specimens, there are two drag régimes: a first low-velocity range where the drag scales as the velocity squared and a second régime for higher velocities in which the drag is almost independent of the velocity with an associated exponent between 0 and 0.2 . In this high-velocity range, the ribbon may be qualitatively described by a horizontal segment and a curved circle arc (see figure 2 for a snapshot of the trapezoidal ribbon). 


\section{T. Barois and E. de Langre}

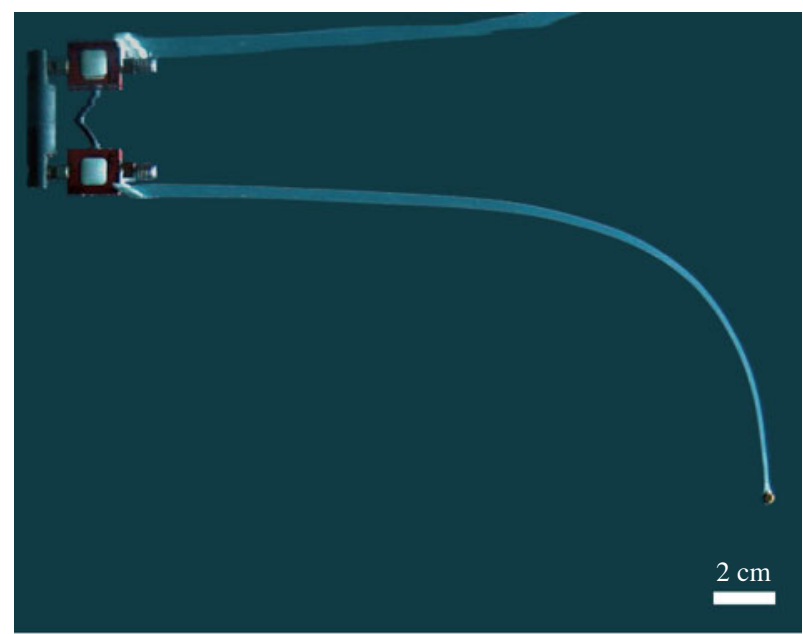

(a)

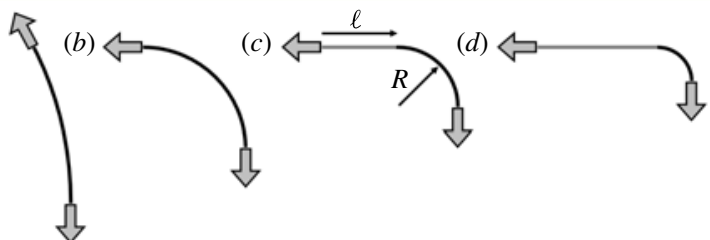

FIGURE 2. Trapezoidal ribbon profile snapshot $\left(U=7.7 \mathrm{~cm} \mathrm{~s}^{-1}\right)$. The top of the snapshot corresponds to the reflection of the ribbon on the water free surface. The schematics at the bottom show the ribbon profile following the simplified model for the pressure drag. For low velocities $\left(a, C_{G} \ll 1\right)$, the ribbon is a circular arc with a radius much larger than its length. For the critical velocity $\left(b, C_{G}=\pi / 2 c\right)$, the ribbon is a quarter-circle. For higher velocities $\left(c, d, C_{G}>\pi / 2 c\right)$ the quarter-circle decreases in size and it is connected with a horizontal segment.

The residual velocity dependence in the high-velocity régime may be attributed either to the drag of the weight at the free end or to the skin friction drag, associated with tangential forces.

\section{Theoretical model}

\subsection{Equation for weighted ribbons in a flow}

The origin of the constant drag comes from the fact that the water flow is acting on the ribbon only by re-orienting the in-plane tension. Consequently, the drag force corresponds to the horizontal component of the tension at the clamped end. As the tension magnitude $T$ is constant, it equals the weight of the mass added at the free end $T=m g$. To obtain the projection of $T$ at the clamped end, it is necessary to consider the equation governing the equilibrium of the ribbon without flexural rigidity. The equilibrium for normal and tangential forces is written $\mathrm{d} T(s) / \mathrm{d} s=f_{t}$ and $T \mathrm{~d} \theta / \mathrm{d} s=f_{n}$ where $s$ is the curvilinear coordinate along the ribbon and $f_{n}$ and $f_{t}$ are the normal and tangential forces per unit length respectively (Landau et al. 1986). If the flow-induced normal forces are indeed dominant for ribbons, $f_{n}$ and $f_{t}$ may be approximated by $f_{t} \sim 0$ with $f_{n}=c(\theta) \rho \mathscr{W}(s) U^{2} / 2$ where $\rho$ is the fluid density, $U$ the fluid velocity with respect to the ribbon, $\mathscr{W}(s)$ the local width of the ribbon and $c(\theta)$ a local drag 


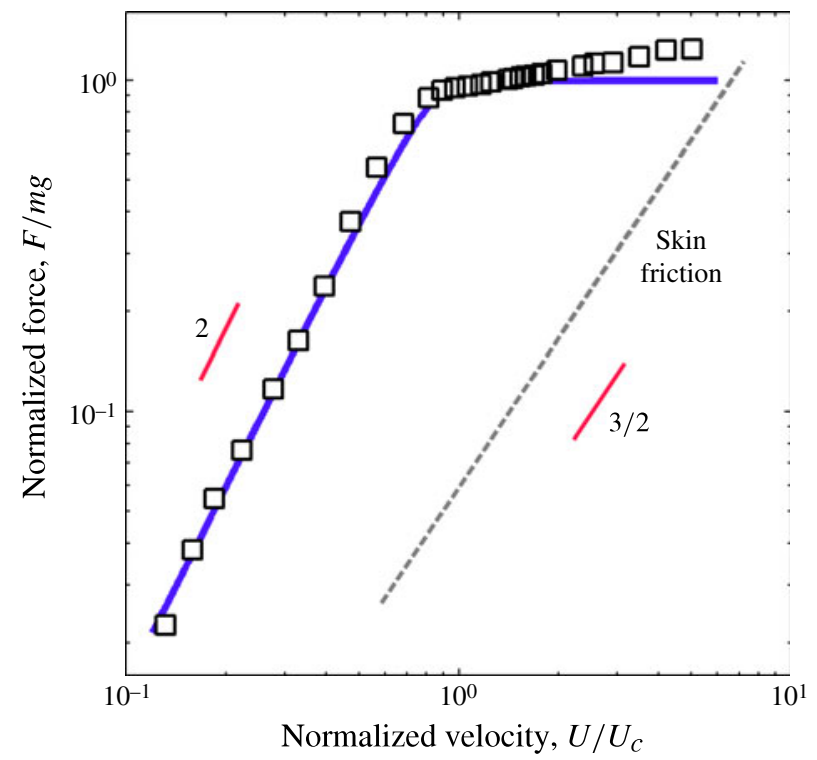

FIGURE 3. Log-log plot of the measured drag versus velocity for a rectangular ribbon (square symbols). The drag is normalized with respect to the weight in water and the velocity with respect to the critical velocity $U_{c}=\sqrt{\pi m g / c \rho W L}$. The solid line corresponds to the simplified model for the pressure drag where the normal force $f_{n}$ is independent of $\theta(c=1.5)$. The skin friction component of the drag is represented by a dashed line with a slope $3 / 2$.

coefficient of order unity that should depend on the local ribbon orientation with respect to fluid velocity. According to these assumptions, the ribbon profile satisfies:

$$
\frac{\mathrm{d} \theta}{\mathrm{d} \tilde{s}}=\frac{1}{2} \frac{\rho L W U^{2}}{m g} w(\tilde{s}) c(\theta),
$$

where a typical width $W$ has been defined for the ribbon $\mathscr{W}(\tilde{s})=W w(\tilde{s})$ with $\tilde{s}=s / L$. The equilibrium shape is then governed by a unique dimensionless number

$$
C_{G}=\frac{\rho L W U^{2}}{2 m g} .
$$

This parameter defined here for a weighted ribbon is similar to the classical Cauchy number for elastic reconfiguration, except that the elastic forces are replaced by the weight. Equation (3.1) may be integrated in principle, knowing the explicit form of the dimensionless width $w(\tilde{s})$, the local drag coefficient $c(\theta)$ and the boundary condition imposed by the weight. From this solution, it is possible to obtain the drag force $F=T \cos [\theta(0)]$. When $C_{G}$ is high enough, $\theta(0)$ is expected to go to zero, which means that the force saturates at level given by the weight as seen in figure 3 . This approach is illustrated using a simplified model presented below.

\subsection{Simplified model of the pressure drag}

For simplicity, the pressure drag component $f_{n}$ is assumed to be independent of the local orientation of the membrane with respect to the fluid velocity. A solution for the profile may then be obtained when the local drag coefficient $c$ is independent of $\theta$. While this model is rather simplified, the experimental data for the rectangular 


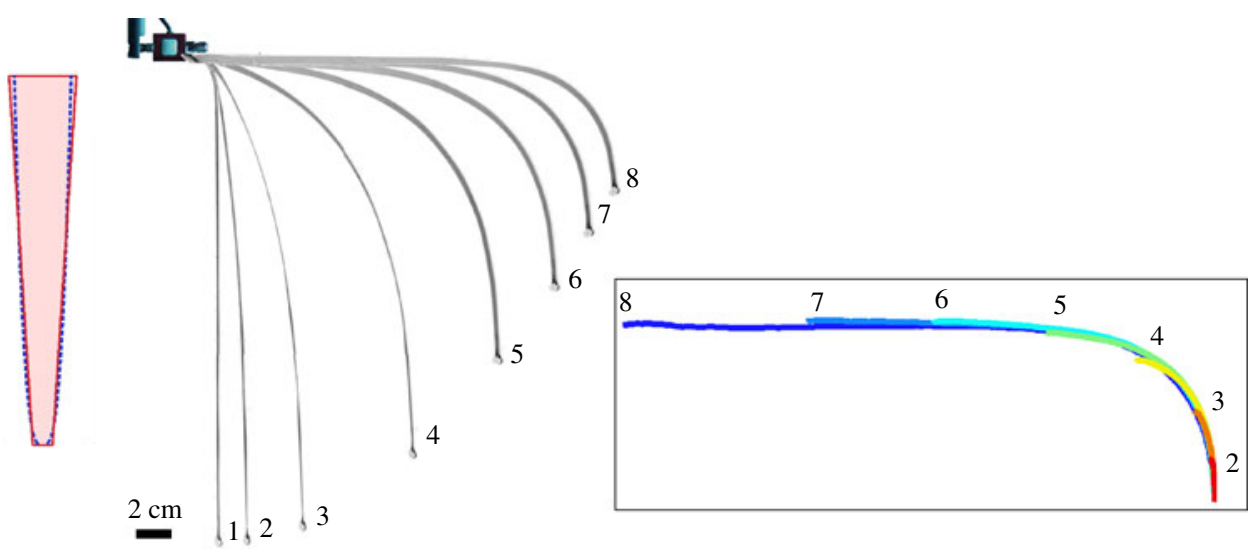

FIGURE 4. Trapezoidal ribbon profile snapshots for varying velocities (ribbon profiles 1-8 for the corresponding velocities $U=0,1.6,2.7,4.1,5.5,6.8,8.2$ and $10.0 \mathrm{~cm} \mathrm{~s}^{-1}$ ). The left inset gives a schematic view of the ribbon geometry (solid red line) which is compared with the width function $w(\tilde{s}) \sim \tilde{s}^{\nu_{w}}$ (dashed blue line, $v_{w}=0.31$ ) used for the self-similar analysis. In the right inset, a plot of the scaled ribbon profiles is represented in scaled coordinates $\left\{X_{s}, Y_{s}\right\}=\{X, Y\}^{1.3} \times C_{G}$.

ribbon $(w(\tilde{s})=1)$ are quantitatively reproduced in figure 3 with $c=1.5$ as a unique fitting parameter. Indeed, the integration of (3.1) is straightforward and the profile is a circular arc

$$
\theta(\tilde{s})=-\frac{\pi}{2}+c C_{G}(1-\tilde{s})
$$

where the boundary condition $\theta(1)=-\pi / 2$ prevails at the free end. For low velocities $\left(C_{G}<\pi / 2 c\right)$, the angle at the clamped end is $\theta(0)=c C_{G}-\pi / 2$, which means that the drag force is $F=m g \sin \left(c C_{G}\right)$. When $C_{G}$ reaches the critical value $\pi / 2 c$, i.e. when the critical velocity $U_{c}=\sqrt{\pi m g / c \rho W L}$ is reached, the ribbon profile takes the form of a quarter-circle. When $C_{G}>\pi / 2 c$, the quarter-circle radius $R=L / c C_{G}$ decreases in size. The clamped end is reached via a horizontal segment of length $\ell=L\left(1-\pi / 2 c C_{G}\right)$ that is parallel to the flow (see the sketch at the bottom of figure 2). The model captures the qualitative features of the trapezoidal ribbon profile during reconfiguration as seen in figures 2 and 4 for different flow velocities, i.e. different $C_{G}$ values.

\section{Ribbon profile self-similarity}

As already mentioned in previous work on reconfiguration (Alben et al. 2002), the existence of a scaling law for the drag may be attributed to the self-similarity of the deformable body. In the following, a trapezoidal ribbon is considered because, in contrast to a rectangular ribbon, it is not subjected to flutter instabilities at high velocities. As seen on the right of figure 4, the experimental profile for the trapezoidal ribbon, remarkably, becomes superimposed on a single master curve if the spatial coordinates $X$ and $Y$ are rescaled according to $\left\{X_{s}, Y_{s}\right\}=\{X, Y\}^{1.3} \times C_{G}$. This rescaling exponent 1.3 may be justified from the profile of the trapezoidal ribbon that can be approximated by $w(\tilde{s})=\lambda \tilde{s}^{\alpha}$, with $\alpha=0.3$ (left inset in figure 4). Using the transformation $\tilde{s}_{s}=\tilde{s}^{\alpha+1} C_{G}$, with $\alpha+1=1.3$, (3.1) takes the form $\mathrm{d} \theta / \mathrm{d} \tilde{s}_{s}=\lambda c /(\alpha+1)$, which defines the collapsing curve in the right part of figure 4. It is important to 


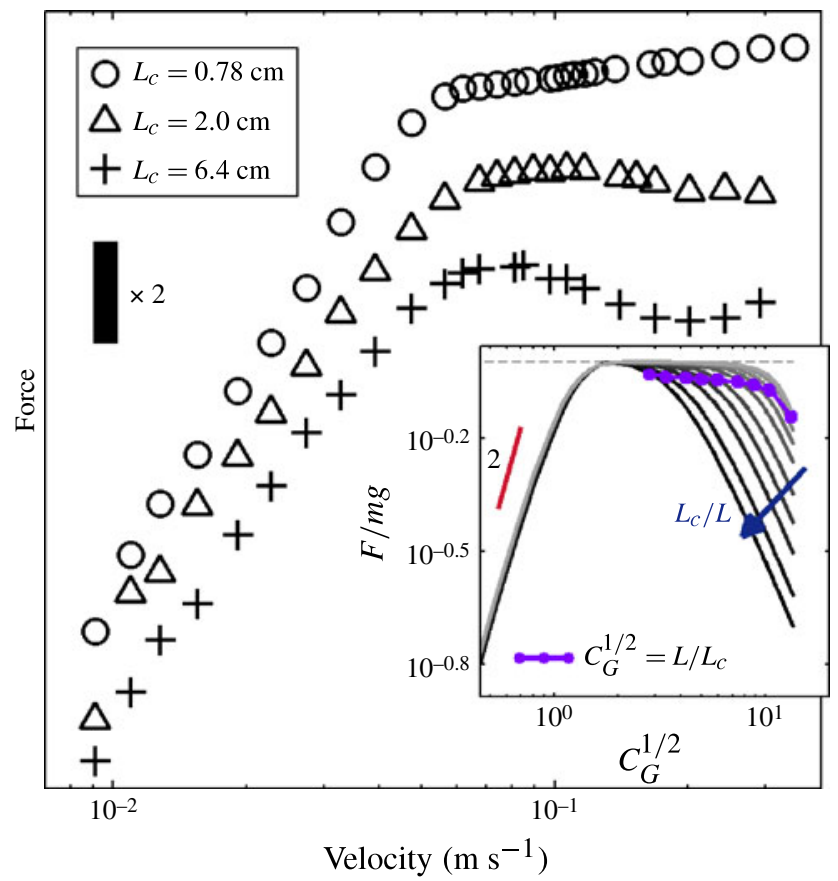

FIGURE 5. Log-log plot of the drag versus velocity measurements for three rectangular ribbons with different bending stiffnesses, i.e. different values for the critical length $L_{c}$ (see text). If $L_{c}$ is not negligible compared to the ribbon length $L=29 \mathrm{~cm}$, the drag decreases with increasing flow velocity. The data are vertically shifted for clarity. The vertical bar on the left corresponds to a factor two for any of the curves. Inset: numerical simulation of the normalized drag $(F / m g)$ as a function of the normalized velocity $\left(U / U_{c}\right)$ for ribbons with critical lengths $L_{c} / L=0.3,0.6$, $0.9,1.3,1.8,2.9,4.1,5.8,9.1$ and $13 \% ; c(\theta)=\sin \theta$. The connected dots represent the onset $U / U_{c}=L_{c} / L$ where the bending stiffness effects become dominant.

note that the equation of the master curve is independent of $C_{G}$ thereby demonstrating self-similarity.

\section{Bending stiffness effects}

In the previous approach, the ribbons have been considered as membranes and therefore bending stiffness has been entirely neglected. For small radius of curvature $R$ (high $C_{G}$ ), the finite value of the bending rigidity $E I$ is no longer negligible as the bending energy scales as $E I / R$. When this energy balances the gravity potential, a critical length $L_{c}=\sqrt{3 E I / m g}$ may be defined. It should be noted that for the drag measurements of figure 1(b), the bending stiffness was indeed negligible as $L_{c}$ was much smaller than $L\left(L_{c} / L\right.$ was $2.7 \%$ for the rectangular ribbon and $3.5 \%$ for the trapezoidal ribbon). To illustrate the effect of bending stiffness, the drag was measured for three rectangular ribbons with increasing values of $L_{c}$ (figure 5). It is observed that the bending effects, i.e. $L_{c}$ approaching $L$, cause the drag force to decrease with increasing fluid velocity. This stems from the fact that the bending term acts as a restoring force to make the ribbon parallel to the flow.

In the limit of small bending stiffness, the modification of the drag can be estimated via a perturbation approach by balancing the weight with the pressure and elastic 
forces:

$$
m g \sim \rho U^{2} \mathscr{R} W+\frac{E I}{\mathscr{R}^{2}},
$$

where $\mathscr{R}$ corresponds to the characteristic size of the curved region of the ribbon that appears in the drag $F \sim \rho \mathscr{R} W U^{2}$. The small-stiffness limit corresponds to $E I / \mathscr{R}^{2} \ll m g$. Let $\mathscr{R}=\mathscr{R}_{0}+\delta \mathscr{R}$. At leading-order when $E I$ is negligible, one obtains $\mathscr{R}_{0} \sim m g \rho^{-1} W^{-1} U^{-2}$, which corresponds to the constant-drag régime $F_{0} \sim m g$. At the next order the perturbation term is given by $\delta \mathscr{R}=-E I R_{0}{ }^{-2} \rho^{-1} U^{-2} W^{-1}$. The drag force may then be expressed as $F \sim m g\left[1-(1 / 3)\left(L_{c} / L\right)^{2} C_{G}{ }^{2}\right]$. This scaling means that the constant-drag régime is valid as long as $C_{G} \ll L / L_{c}$. If $C_{G} \sim L / L_{c}$, the bending stiffness is non-negligible and it lowers the drag. To describe more accurately the drag when the bending term $E I / \mathscr{R}^{2}$ is no longer small, a numerical solution of the ribbon profile for varying velocity and stiffness is necessary (inset in figure 5). The angular dependence of the local drag coefficient, $c(\theta)=\sin \theta$, is selected in order to smooth out the discontinuity in the normal force at the connection point between curved and straight portions of the ribbon. The simulation shows that the bending effects appear when $C_{G} \sim L / L_{c}$ (connected dots in the inset of figure 5). When $C_{G}>L / L_{c}$, there is an asymptotic régime for the drag dependence on velocity: the velocity exponent is found to become negative and its magnitude depends on the function $c(\theta)$.

\section{Conclusion and perspectives}

The existence of a constant-drag régime has been demonstrated for a range of velocities for ribbon membranes. The number $C_{G}$ that measures the relative importance of the drag for the equivalent rigid body and the weight is the appropriate dimensionless number of the problem. A constant-drag régime has been identified for the range of velocities corresponding to $1<C_{G}<L / L_{c}$ where the lower bound signals the appearance of a straight horizontal ribbon segment and the upper bound corresponds to the appearance of bending stiffness effects. This $C_{G}$ interval is typically observed as the flow velocity is increased.

The constant-drag concept presented here is based on the fact that ribbon membranes transmit only tangential forces. In the pressure drag régime, fluid forces modify the orientation of the in-plane tension but not its magnitude. For this reason, the effect of the fluid on the ribbon orientation may be compared to the effect of a pulley on a string under tension.

It may be argued that this entire study completely neglects the skin friction drag component $f_{t}$. An estimation of the skin friction drag is displayed in figure 3 . It is seen that the skin friction drag might be the origin of the residual increase of the drag with velocity. This skin friction drag is then a limiting factor for the validity of the present analysis.

A decrease of the drag with velocity was observed when the ribbon has a significant flexural rigidity. The experiments, the low-flexural-rigidity perturbation approach and numerical simulations together demonstrate that the velocity dependence of the drag may be tuned by adjusting the bending stiffness of the ribbon. This drag tuning may be effectively used, for instance, to compensate any additional drag of the structure. In the perspective of potential applications, this observation is of great interest as it means that such negative differential drag may be appropriately combined with non-deformable bodies in order to obtain an effective cancellation of the velocity dependence. 


\section{Acknowledgements}

The authors gratefully thank L. Tadrist, C. Clanet and more specifically P. Huerre for their careful reading of the manuscript. This work was supported by the funding program DGA-2041.

\section{References}

Alben, S., Shelley, M. \& Zhang, J. 2002 Drag reduction through self-similar bending of a flexible body. Nature 420, 479.

Albert, R., Pfeifer, M. A., Barabási, A.-L. \& Schiffer, P. 1999 Slow drag in a granular medium. Phys. Rev. Lett. 82, 205.

Blevins, R. D. 2003 Applied Fluid Dynamics Handbook. Krieger.

EnNos, A. R. 1997 Wind as an ecological factor. Trends Ecol. Evol. 12, 108.

Gosselin, F., DE LAngre, E. \& Machado-Almeida, B. A. 2010 Drag reduction of flexible plates by reconfiguration. J. Fluid Mech. 650, 319.

Harder, D. L., Speck, O., Hurd, C. L. \& SPeCK, T. 2004 Reconfiguration as a prerequisite for survival in highly unstable flow-dominated habitats. J. Plant Growth Regul. 23, 98.

Landau, L. D., Lifshitz, E. M., Kosevitch, A. M. \& PitaevskiĬ, L. P. 1986 Theory of Elasticity 7. Butterworth-Heinemann.

De Langre, E. 2008 Effects of winds on plants. Annu. Rev. Fluid Mech. 40, 141.

LUHAR, M. \& NEPF, H. M. 2011 Flow-induced reconfiguration of buoyant and flexible aquatic vegetation. Limnol. Oceanogr. 56, 2003.

SAND-JENSEN, K. 2003 Drag and reconfiguration of freshwater macrophytes. Freshwat. Biol. 48, 271.

Schouveiler, L. \& Boudaoud, A. 2006 The rolling up of sheets in a steady flow. J. Fluid Mech. $563,71$.

Statzner, B., Lamouroux, N., Nikora, V. \& Sagnes, P. 2006 The debate about drag and reconfiguration of freshwater macrophytes: comparing results obtained by three recently discussed approaches. Freshwat. Biol. 51, 2173.

Vogel, S. 1996 Life in Moving Fluids. Princetown University Press.

Vogel, S. 2009 Leaves in the lowest and highest winds: temperature, force and shape. New Phytol. 183, 13.

WIRTZ, D. 1995 Direct measurement of the transport properties of a single DNA molecule. Phys. Rev. Lett. 75, 2436. 